\title{
Programming service tests with TTCN
}

\author{
Martin Anlauf \\ Bosch Telecom GmbH \\ Kleyerstraße 94, D-60326 Frankfurt am Main, Germany \\ martin.anlauf@fr.bosch.de \\ tel: +496975056148 fax: +496975056168
}

\begin{abstract}
This paper describes an experiment in which TTCN is used to program service test cases for a private switch. One goal of the experiment was to evaluate the suitability of TTCN for another context than conformance testing. The requirements of the experiment and the nature of the test cases under consideration are described. The importance of concurrency is emphasized. The methodology of development is outlined, including the derivation of test purposes from verbal descriptions. The test method is indicated. Concepts directing the design of the test suite are introduced. Experiences and conclusions are presented.
\end{abstract}

Service Test, TTCN, ISDN

\section{Keywords}

\section{INTRODUCTION}

The experiences described in the paper result from an experiment run at Bosch Telecom GmbH. The suitability of TTCN is evaluated to improve the testing phase in the development process for private communication switches. The functionality under focus is that of ISDN layer 3 protocol DSS1 (ETS 95).

The introduction describes requirements and goals of the experiment. Section 2 discusses the nature of the considered test cases. Section 3 presents the test method as well as concepts which have directed the design of the test suite. Section 4 describes experiences. Section 5 presents concluding results.

\subsection{Current test practise and requirements}

The peculiarities of the described TTCN application in contrast to pure conformance test shall be clarified first. To this purpose, the current test practise for private switches is outlined and emerging requirements are sketched. 
When a new software release is integrated on the target hardware a regression test phase is entered. In this phase, a number of selected test cases is executed to make sure that the functionality of the previous versions is still valid. Only when the release has passed the regression test, more extensive tests are executed. If the regression test is not passed, it has to be repeated after repair of the release.

Regression test cases are carried out by hand once and recorded. Basically they can be executed automatically the next time. In an automatic execution, the test machine sends recorded stimuli to the switch and compares the received reactions to the recorded reactions. If theses reactions are identical then the verdict pass is given. Else the test case is regarded as failed.

A new software release may cause some changes in the behaviour of the switch, i.e. in reactions to given stimuli. Such changes range from additional information elements inside of protocol messages to the sending of different protocol messages. In such cases, the automatic execution of test cases may lead to the verdict fail, even if these changes are conformant to the standard. This means that the manual execution and the recording of a large number of test cases may have to be repeated, which costs time and effort.

\subsection{The experiment}

The goal in programming test cases is to save time and effort in the regression test phase. The test cases should be automatically executable but more robust with regard to those changes in behaviour which remain within the limits of the standard. If repeated manual execution of test cases can be avoided, the effort spent to develop a test suite should be regained.

The considered test cases are estimated to belong to service testing, in contrast to conformance testing. This is because not the protocol conformance of the switch is focused. The functioning and interaction of integral communication services are checked. However, in our approach of service testing the exchanged protocol messages are examined, and it is difficult to define a clear distinction to conformance testing.

TTCN is a standardized notation (ISO 97). It is designed to be used in conformance testing (ISO 94). The suitability of TTCN for other testing contexts remains to be evaluated. This paper describes an application in service testing.

The evaluation of this TTCN application was done in the RESTATE project. RESTATE was funded by the European Systems and Software Initiative (ESSI) as a Process Improvement Experiment (PIE), ESSI project no. 23978. RESTATE started in March 1997 for a duration of 18 months.

The project was accompanied by a measurement program. The Goal-QuestionMetrics method (GQM) was applied to measure efforts and benefits of the TTCN employment. Since at the time of edition of the paper there were no 
representative results of the measurement program available, they will be presented on slides.

The paper does concentrate on design aspects. For a more complete description of the RESTATE project (Münzel 97).

\section{THE TEST CASES TO CODE}

The first subsection gives an example of a service test case. The second outlines some characteristics of the test cases. The following subsections discuss the task to do and sketch the test environment.

\subsection{An example}

The test cases of the regression test are currently executed manually by humans, and also the described actions and reactions belong to the level of perception of a human. The test cases are described in an informal and verbal way. To give a flavour of such a description, an example is presented.

\section{Test Conditions:}

Subscriber B has no knocking protection

\section{Actions:}

a) Subscriber A takes the handset off hook

Subscriber A dials all digits

b) Subscriber B takes the handset off hook

c) Subscriber A puts the handset on hook

d) Subscriber B puts the handset on hook

\section{Reactions:}

a) Terminal $B$ is ringing

b) Subscribers A and B have connection

c) Subscriber B hears busy tone Subscriber A has initial state

d) Subscriber B has initial state

There are also more complex test cases involving more subscribers, some subscribers handling several calls.

\subsection{Characteristics}

Some characteristics of the test cases under consideration are pointed out.

- Concurrency Several parties are acting concurrently. 
- Dependencies There are dependencies between the actions of the different parties. Some dependencies are synchronized implicitly by the switch and do not have to be modelled explicitly. For example it is supposed that terminal $\mathrm{B}$ rings only after subscriber $\mathrm{A}$ has dialed the number of $\mathrm{B}$. Other dependencies have to be synchronized explicitly. An example would be when in the presented test case a third subscriber $C$ would also want to call B, but only after A and B have established a connection.

- Configuration A certain configuration of the switch may be a precondition to execute a test case. For example a subscriber is authorised to use a certain service or not. If several test cases are executed in a series automatically, then such configurations of the switch may have to be done automatically as well.

- Internal values For some test cases it is necessary to check internal parameter values of the switch. For example a test case could have to check the correct accounting of charges. Such checks of internal values should be automated as well.

It is to be noted that the last two points leave the realm of pure black box testing. Further the configuration of the switch and the check of internal values can be done via a proprierary protocol. The aspects concerning the configuration of the switch and the check of internal values are not covered in the paper.

\subsection{The task}

In the experiment there are considered 33 cases to test the basic functionality "basic call" of the switch. Additionally the functionality of supplementary service "Advice of charge" is checked by 17 test cases. These 50 test cases represent together only a small part of the regression test.

The task is to take the given verbal descriptions of the test cases and to code them in TTCN. There is work to be done in two respects.

- Bridging the gap between the levels of abstraction The verbal descriptions represent the human point of view. The message flow on the protocol interface has to be derived from the sequences of actions and reactions. For example the action take handset off hook may correspond to the sending of a Setup message and the receipt of a Setup Acknowledge. The flow of the messages represents the "protocol point of view".

- Refinement of the descriptions To obtain a complete TTCN test suite, there is more information to be added. Declarations and constraints are needed. The Messages to be sent or received have to be specified more precisely, up to the values of contained information elements. 


\subsection{The test environment}

The test cases are executed on a A8619 from Alcatel/Sel. It is connected to the switch with $2 \mathrm{~S}_{0}$ and $2 \mathrm{UP}_{0}$ ports. The 4 ports can be used either as 4 subscribers or as 3 subscribers and 1 system console. The system console is used to configure the switch and to check internal values.

The test machine is running the TTCN Professional software package from Expert Telecoms. The package includes an editor, a compiler, a test campaign manager, a simulator for the lower layers as well as a tool supporting the analysis of recorded test runs.

The development of the test suites is done with the Concerto TTCN editor from Sema Group, which is running on Sun workstations. The test environment is sketched in Figure 1.

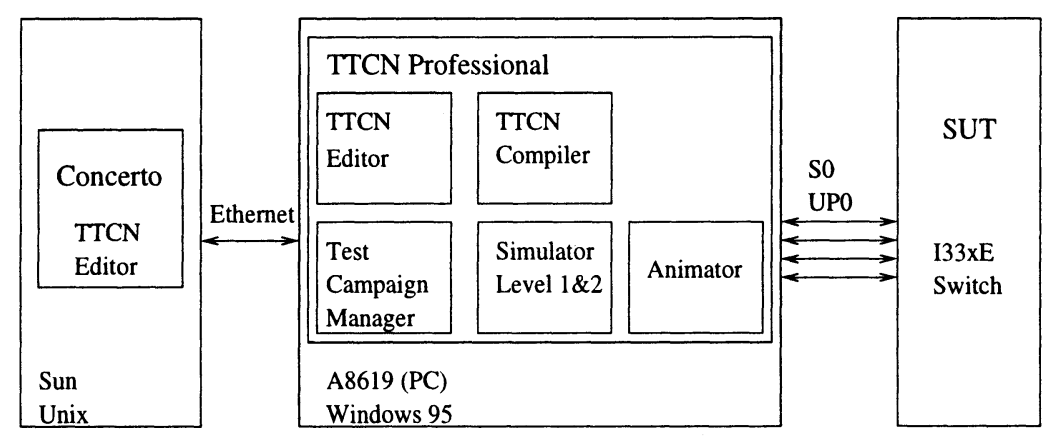

Figure 1 Test Environment

\section{CONCEPTS}

This section introduces concepts and design decisions. It presents the applied test method and sketches the test case configurations. A model of the user's behaviour is introduced which directs the structure of the test step library. Some of the conventions applied are mentioned and the use of message flow diagrams is illustrated.

\subsection{The test method}

A multi party test method is applied which is sketched in Figure 2. Among the four classical single party test methods, it has the closest resemblance to the remote test method (ISO 94).

There are several testing parties involved, all lower testers running on the same test machine. The exact number of testing parties depends on the test 


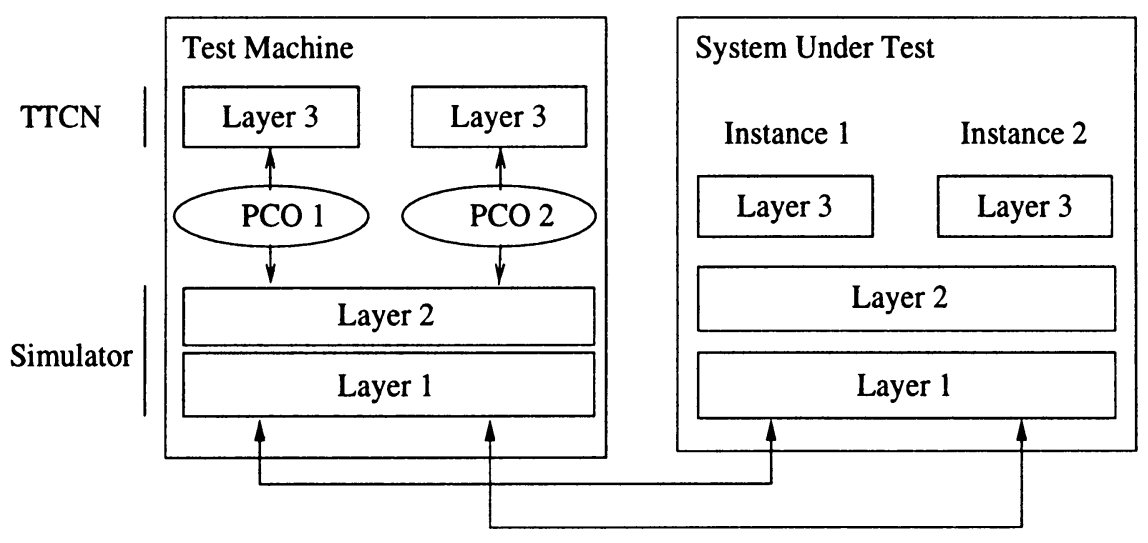

Figure 2 Multi party test method. Illustration with 2 testing parties.

case. There are no upper testers. The TTCN description of a test case specifies the layer 3 behaviour of all participating testing parties. The lower levels are simulated by the test machine. Each testing party is realized as a separate parallel test component (PTC). The control of the testing parties is realized with coordination messages from the master test component (MTC).

Each testing party has a corresponding instance of a protocol state machine on the switch. The test purposes do not only aim at the correct behaviour of such a single instance. The interaction between several instances is also important to assure the services of the switch.

\subsection{The test case configurations}

The configurations used by the test cases all have the same structure. They only differ in the number of participating PTCs. Figure 3 shows an example.

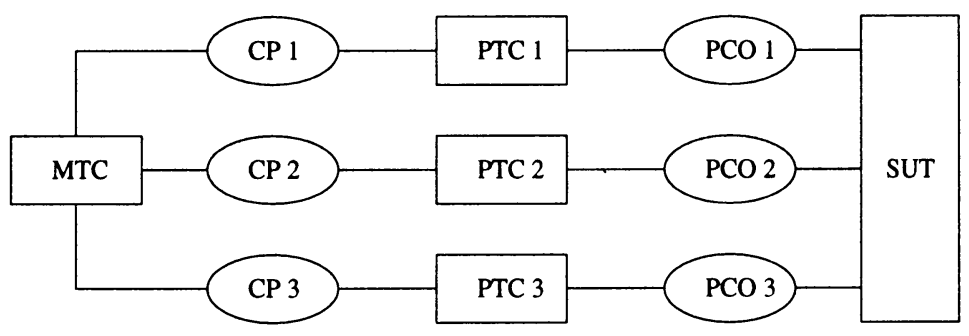

Figure 3 Configuration of test cases. Example with 3 PTCs.

Note that the MTC does not communicate directly with the SUT. The whole communication with the SUT is done by the PTCs via the points of 
control and observation (PCOs). On the other side the PTCs do not have the possibility to communicate with each other directly. All coordination is done by the MTC via the coordination points (CPs).

The simplicity of the configurations allow a uniform coordination of the PTCs in all test cases, no matter how many PTCs are involved. The coordination is separated from the communication with the SUT. Further the communication with the SUT is divided into smaller parts, which are easier to handle. Each PTC only has to know it's own part of the communication.

\subsection{A user model and the test step library}

The verbal descriptions of the test cases only use a small number of actions and reactions. Actions and reactions are subsumed as events. Examples are $X$ takes handset off hook, $Y$ dials some numbers or $Z$ is ringing. These events reflect the perception of the human user. With TTCN in contrast, the message flow on the protocol interfaces has to be described.

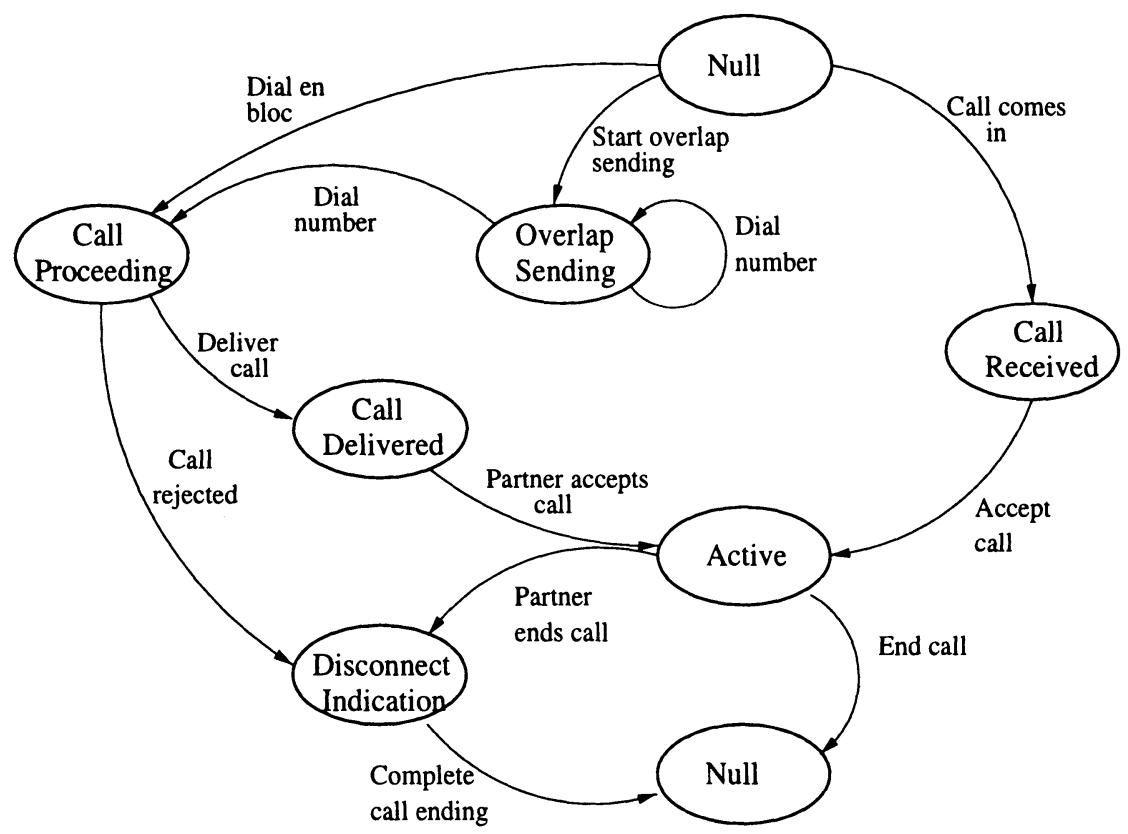

Figure 4 A model of the user's behaviour

The idea now is to model the behaviour of the user in order to fix all possible events. When it is known which flow of protocol messages corresponds to which event, then the events can be coded as test steps. When such a test step is parameterized in a suitable way, then it can be used by different PTCs. 
In this style a test step library is built which hides details of the protocol and which reflects the perception of the human user. The test step library is meant to constitute a set of components from which test cases can easily be constructed.

The model of the user's behaviour is sketched in Figure 4 in the style of a state transition diagram. The diagram is not complete and should not be interpreted in a formal way. It simply did serve as a starting point to design the test step library. Each transition is realized as a test step.

The events in our example concerning subscriber A, i.e. Take handset off hook, dial all digits, $A$ and $B$ have connection etc. correspond in the diagram to the transitions Start overlap sending, Dial number, Partner accepts call etc. The events concerning subscriber B, i.e. Terminal is ringing, take handset off hook etc. correspond to the transitions Call comes in, Accept call etc.

\subsection{Conventions}

In order to maintain a uniform style in the test suite, a set of conventions was applied. The conventions range from a conceptual to a syntactical level. Conceptual conventions describe the test case configurations, the coordination of test components and the error treatment. The issues of default behaviour and of verdict passing are also addressed. The syntactical conventions cover aspects like names and parameters of test steps and constraints. TTCN style rules are also defined.

Since concurrency is a crucial point of the project and the number of participating testing parties should not be restricted a priori, special effort has been put into the conventions to coordinate the test components. Two cases of coordination are distinguished.

- Synchronization Synchronization is necessary in the case when one component is allowed to perform an action only when some other components have fulfiled some conditions.

- Error treatment When one component detects an error which leads to the termination of the test case, all other components have to be informed about the forthcoming termination in order to clear their connections.

The test suite writer knows the points in the test case where components have to be synchronized. So the coordination for synchronization is described explicitly in the dynamic tables of the test case. On the other hand it is not predictable at which point errors will be detected. So the coordination in the error case is described and "hidden" in defaults.

The approach in the project is to fix a small number of coordination messages and to restrict their use, in order to get a uniform coordination style.

The descriptions of the behaviour of the PTCs of a test is divided into 3 classical parts: preamble, body and postamble. The preamble may contain test steps to configure the SUT as required and to bring it into a suited starting 
state. The body contains the test steps of the library mentioned before. The postamble may contain steps to check internal values, to reconfigure the SUT and to bring it into a suited final state.

\subsection{Message flow diagrams}

When concurrency is to be described, the most important thing is to keep the overview of the expected behaviour or communication. Here a description of the expected flow of messages on several intefaces is needed. There may not be determined a total ordering on the messages a priori.

For example when two messages are expected at two different interfaces, one message at each interface, the ordering of arrival may not be predictable. So there is only a partial ordering defined on the message flow.

While the presented verbal descriptions of the test cases do not even mention protocol messages, the TTCN code is very detailed and difficult to read. So the need for an intermediate notation arises, which concentrates on the message flow. Following the terminology of the ISO (ISO 94) such descriptions can be considered as test purposes.

Message flòw diagrams are an appropriate notation for this purpose. Although the standardized notation of message sequence charts (MSCs) fulfils all the requirements (ITU 97), in the project an informal variant of message flow diagrams is used. An example for the (ab-)use of notation in the project is that message boxes are used to indicate hierarchy.

The diagrams indicate the expected message flows of test cases. They also sketch the software structure of a test case. The diagrams are used at two levels. An overview diagram contains all components participating at a test case, i.e. the PTCs and the MTC. In the overview diagram only the used test steps are sketched, not the protocol messages. Then there is a specific diagram for each PTC involved which indicates the test steps used by this PTC as well as the protocol messages to send or to receive.

To illustrate this use, 3 diagrams which refine our example are presented in the appendix.

\section{EXPERIENCES}

This section presents experiences from the project. It covers the subjects tools test case validation, parallel editing and aspects of the notation TTCN.

\subsection{Tools}

The used tools were suitable and fulfiled their purpose. It has turned out that user-friendly tools are crucial for the work with TTCN. Features like syntax and semantic checks are valuable, as well as support for the analysis of test runs. 
Since concurrency constructs have been especially essential in the project it did cause some delay that not all the constructs were supported by the tools from the beginning. The critical tool has been the TTCN compiler.

\subsection{Validation of test cases}

Validation is important to get trustworthy test cases. In the project there was the lucky situation that a reference implementation of the SUT was available. Validation consisted mainly in running the test cases against the reference implementation and in analysing the test runs. Mutual reviewing of test suites is also recommendable.

The software package TTCN Professional contains a tool called Animator which turned out to be very helpful for the analysis of test runs. It offers a debug functionality which allows the user to step through the executed commands of a TTCN test case. Equally important are traces of the runs, which record the exchanged protocol messages and coordination messages.

\subsection{Parallel editing}

It is not easy for several developers to work on the same suite because of the many references. It is important to fix the personal rights to modify the different parts of the suite. Parallel editing is eased by test suite fragmentation. The editor Concerto did support this possibility. For the future, the standardized modular features of TTCN are to be welcomed.

\subsection{The language TTCN}

TTCN is stated to be an "informal notation", but in RESTATE it was used just like any other programming language. A preliminary version of 2 nd edition TTCN (ISO 97) was the basis of the project, but not all new features have been used. Concurrency has been used heavily. Defaults have been very helpful, also the Return statement. It has already been mentioned that modularity has not been used. Proceduraly defined test suite.operations have not been used neither.

The conclusion is that the TTCN is suited to describe test cases not only in the restricted field of conformance test, but also to describe service test cases such as those above. However, some shortcomings have been experienced and the language could still be improved.

- Overview TTCN is not an easy language to read. It is difficult to keep the overview. One reason is that the principle of reference is used extensively. Space is valuable inside the narrow TTCN columns, which leads to a heavy use of references. Another reason is that the tabular style is not always well suited for the dynamic part. A big dynamic table is hard to read, which 
leads to the use of many small tables. In this way the information about dynamics is often spread over many tables.

- Ordering of events It would be nice to have the possibility to relax the strictness of the ordering of events in time. In a situation where $n$ events are being waited for, the exact ordering of these events might not be known. It is cumbersome to write down all $n$ ! possible orderings. It would be nicer to have the possibility to "collect" the events without considering the exact order.

\section{CONCLUSIONS}

This section presents some conclusions. They concern the expectations, a side effect, MSCs and the problem of acceptance. Finally, a summary is given.

\section{$5.1 \quad$ Expectations}

The main expectations of the project have been fulfiled. Namely, it has been possible to code the considered test cases in TTCN so that they are executable automatically. They are also robust with regard to protocol conformant changes of the behaviour of the SUT.

\subsection{A side effect}

A side effect of RESTATE has been to deepen our understanding of the test process for private communication switches and to discern a point of possible improvement. The standard of DSS1 often contains options. An enforcement of the documentation of implementation specific details will ease testing as well as development.

\subsection{Message flow diagrams}

The use of message flow diagrams has been proved worthy. For similar projects, the use of the standardized notation MSC '96 is recommended (ITU 97).

\subsection{Acceptance}

1 RESTATE, the coding of the test cases has been done by a team from sutside the group in whose development process TTCN is thought to be integrated. The main expectations have been fulfiled, but it also has been shown that the effort to code test cases in TTCN is big and that the complexity of the language is high. Therefore it is still not clear how TTCN will be integrated in the development process. 


\subsection{Summary}

Concluding it is stated that it is possible to manually code service test cases in TTCN, but that the effort is high. The introduction of TTCN is considered to be an investment, and it is must be estimated carefully, for which purposes the application of TTCN is justified.

It is an open point wether if the protocol interface is the right one to specify service test cases. An alternative would be to look for an interface on a higher level, where the details of the protocol do not have to be described. The choice of the right interface should depend on the goals of the test phase under consideration.

However, if TTCN is employed and coded manually, the use of an application specific test step library has been proved worthy. The use of MSCs to describe test purposes is recommended.

It would be a tempting alternative to evaluate the possibilities of automatic test case generation out of MSCs as it is described in recent papers, cf. (Ek et al. 97) and (Grabowski et al. 97) in the context of service testing.

\section{ACKNOWLEDGEMENTS}

The author would like to thank all people that supported the RESTATE project, especially to Jörn Münzel, to Bertram Weber and to Helmut Meyer. Further thanks to Andreas Zamperoni for the explanation of diverse tools, to Mark Andrew for reviewing and to the group around Jens Grabowski for fruitful discussions.

\section{GLOSSARY}

$\begin{array}{ll}\text { CP } & \text { Coordination Point } \\ \text { DSS1 } & \text { Digital Subscriber Signalling No. One } \\ \text { ESSI } & \text { European Systems and Software Initiative } \\ \text { GQM } & \text { Goal Question Metrics } \\ \text { ISDN } & \text { Integrated Services Digital Network } \\ \text { MSC } & \text { Message Sequence Chart } \\ \text { MTC } & \text { Master Test Component } \\ \text { PCO } & \text { Point of Control and Observation } \\ \text { PIE } & \text { Process Improvement Experiment } \\ \text { PTC } & \text { Parallel Test Component } \\ \text { SUT } & \text { System Under Test } \\ \text { TTCN } & \text { Tree and Tabular Combined Notation }\end{array}$




\section{REFERENCES}

Ek, Grabowski, Hogrefe, Jerome, Koch and Schmitt 97 Towards the industrial use of validation techniques and automatic test generation methods for SDL Specifications Proc. of the 8th. SDL Forum, Evry, France, ed. Cavalli and Sarma, Elsevier Science,1997.

ETS 95 Integrated Services Digital Network (ISDN); Digital Subscriber Signalling System No. one (Dss1) protocol; Signalling network layer for circuit-mode basic call control. ETS 300 403-1 \& 2. European Telecommunications Standards Institute, Sofia Antipolis, 1995 (ITU-T Recommendation Q.931 (1993), modified).

Grabowski, Scheurer, Dai and Hogrefe 97 Applying SAMSTAG to the B-ISDN protocol SSCOP Proc. of IWTCS 10th., Cheju Island, Korea, ed. Kim, Kang and Hong, Chapman \& Hall, 1997

ISO 94 Information Technology, Open Systems Interconnection, Conformance Testing Methodology and Framework. International Standard IS-9646-1 \& 2. ISO, Geneve, 1994.

ISO 97 Information Technology, Open Systems Interconnection, Conformance Testing Methodology and Framework. International Standard IS-96463, Delivery 9.6. ISO, Geneve, 1997.

ITU 97 ITU-TS Recommendaion Z.120: Message Sequence Charts (MSC 96). ITU-TS, Geneve, 1997.

Münzel 97 Quality Improvement Through Formalised Testing Proc. of CONQUEST 1, Arbeitskreis Software Qualität Franken e.V., Erlangen, Germany, pp. 184-187, 1997.

Prevot, D'Hooge and Labbe 96 Experiences with concurrent TTCN for GSM/SS7 testing Proc. of IWTCS 9th., ed. Baumgarten, Burkhardt and Giessler, Chapman \& Hall, 1996

\section{BIOGRAPHY}

Martin Anlauf graduated in 1994 from the University of Kaiserslautern, where he worked from 1995 to 1996 as assistant. In 1996 he started to work at Bosch Telecom in the software technology department. His interests include testing and formal description techniques.

\section{APPENDIX}

The following diagrams illustrate the flow of messages of the example test case. The use of test steps is indicated with comment boxes. The overview diagram containes all participating parties. The specific diagrams concentrate on only one testing party. 


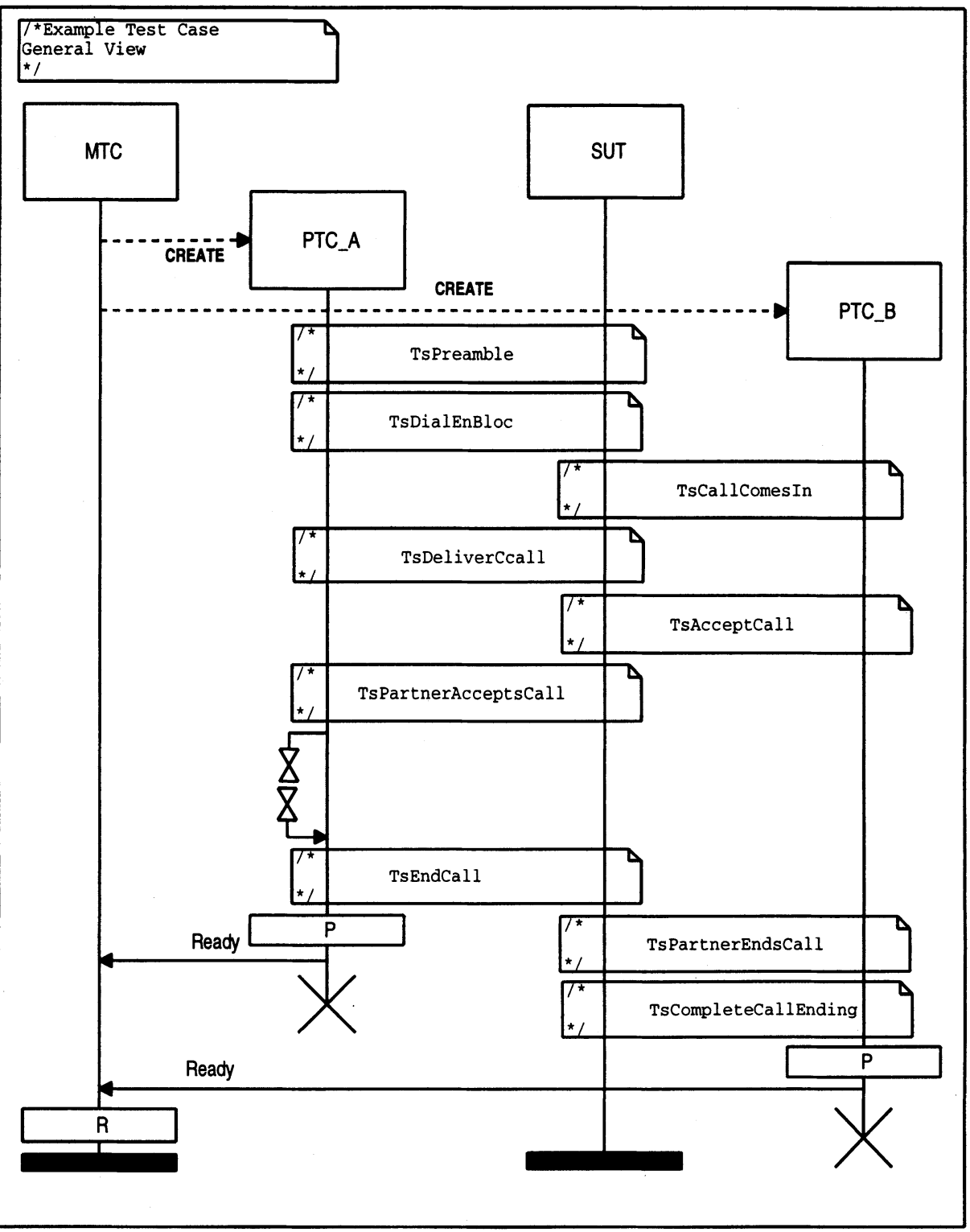

Figure 5 Overview diagram 


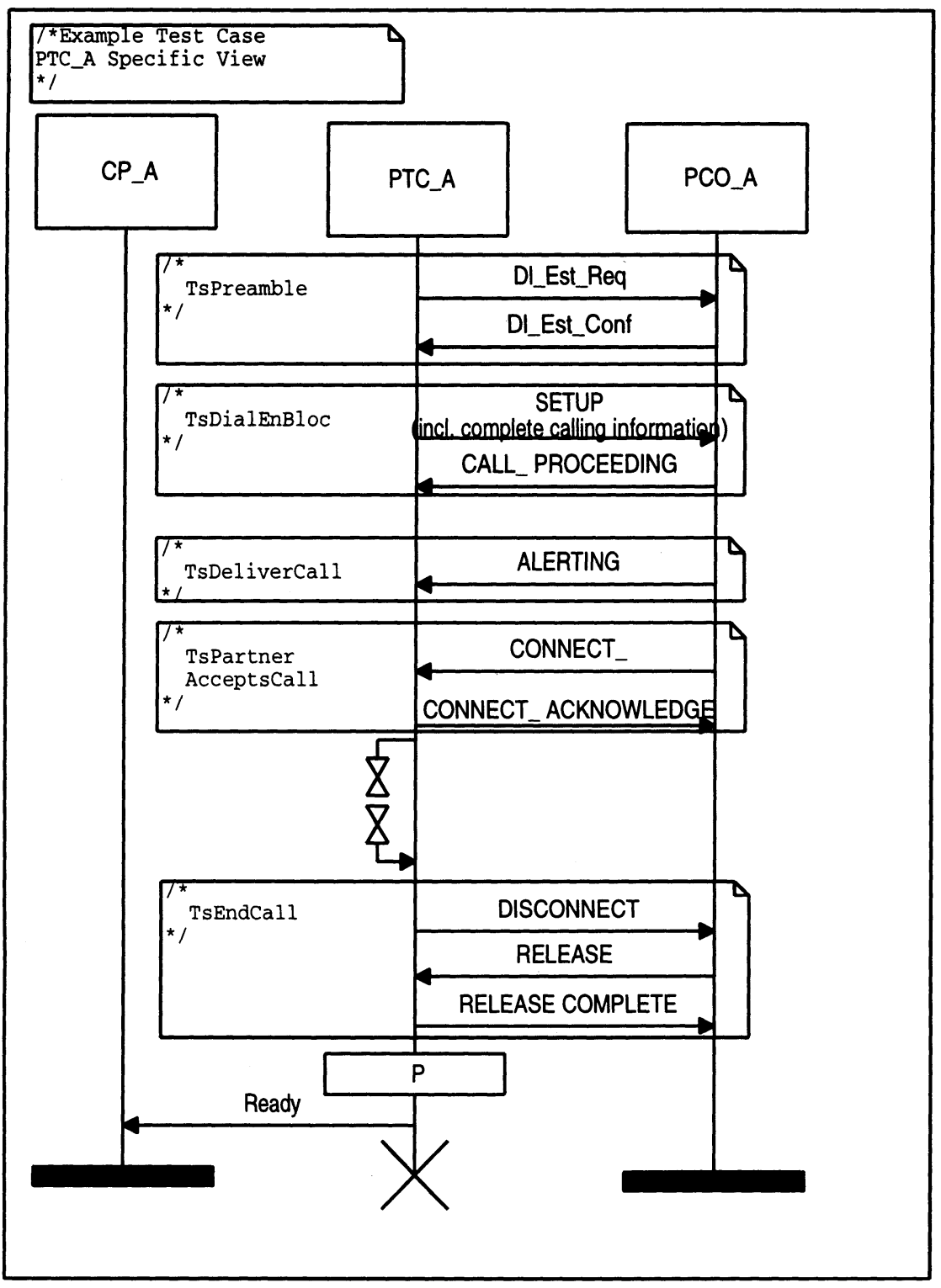

Figure 6 Specific view of subscriber A 


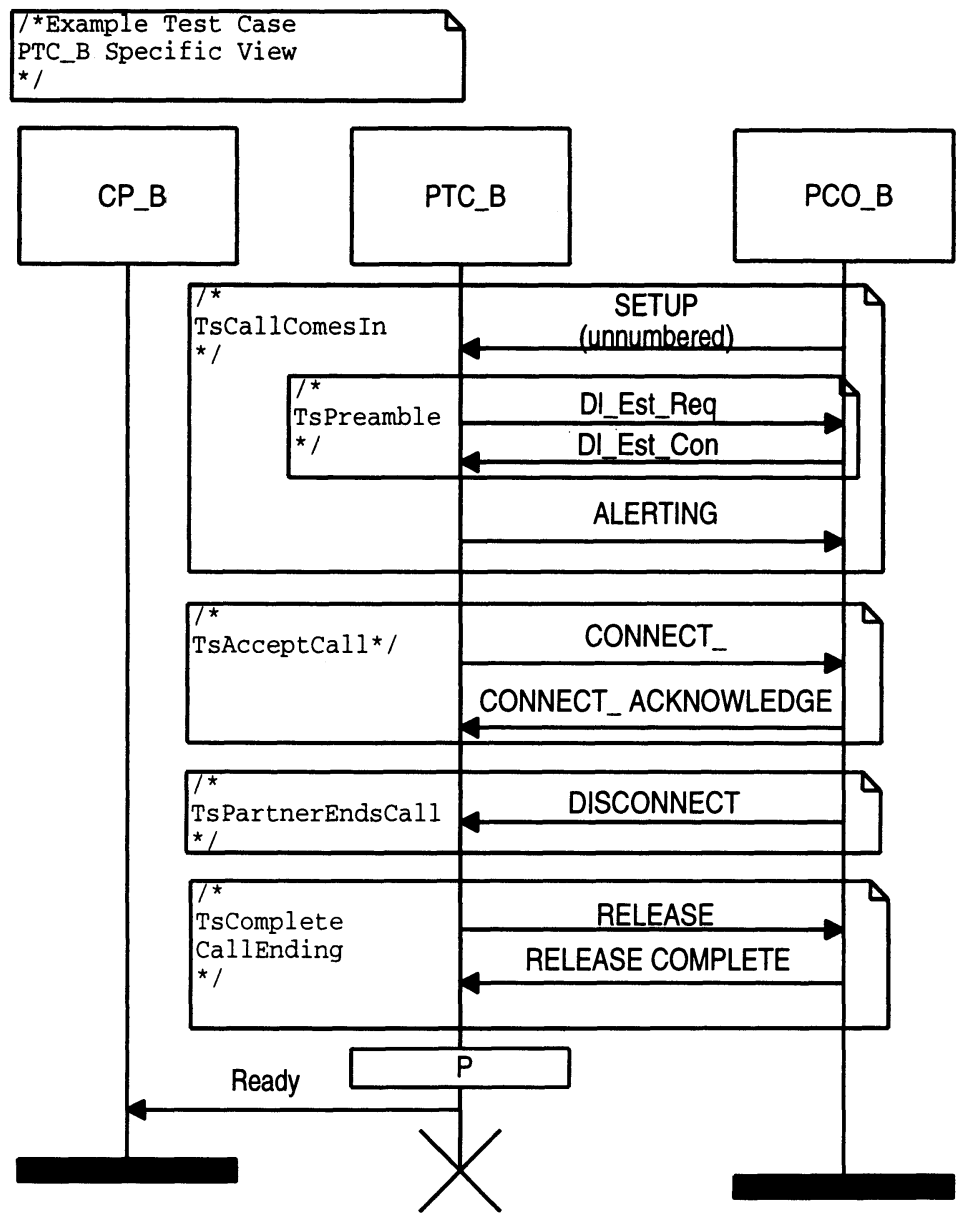

Figure 7 Specific view of subscriber B 